Markert, Jana

\title{
Biografische Selbstreflexion in der Lehramtsausbildung: Erarbeitung der eigenen Bildungsbiografie mittels der systemischen Methode des Lebensfluss-Modells
}

Haushalt in Bildung \& Forschung 8 (2019) 4, S. 118-132

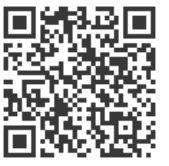

Quellenangabe/ Reference:

Markert, Jana: Biografische Selbstreflexion in der Lehramtsausbildung: Erarbeitung der eigenen

Bildungsbiografie mittels der systemischen Methode des Lebensfluss-Modells - In: Haushalt in Bildung \& Forschung 8 (2019) 4, S. 118-132 - URN: urn:nbn:de:0111-pedocs-236521 - DOI: 10.25656/01:23652

https://nbn-resolving.org/urn:nbn:de:0111-pedocs-236521

https://doi.org/10.25656/01:23652

in Kooperation mit / in cooperation with:

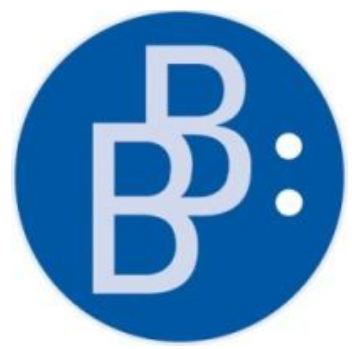

https://www.budrich.de

\section{Nutzungsbedingungen}

Dieses Dokument steht unter folgender Creative Commons-Lizenz: http://creativecommons.org/licenses/by-sa/4.0/deed.de - Sie dürfen das Werk bzw. den Inhalt vervielfältigen, verbreiten und öffentlich zugänglich machen sowie Abwandlungen und Bearbeitungen des Werkes bzw. Inhaltes anfertigen, solange sie den Namen des Autors/Rechteinhabers in der von ihm festgelegten Weise nennen und die daraufhin neu entstandenen Werke bzw. Inhalte nur unter Verwendung von Lizenzbedingungen weitergeben, die mit denen dieses Lizenzvertrags identisch, vergleichbar oder kompatibel sind.

Mit der Verwendung dieses Dokuments erkennen Sie die Nutzungsbedingungen an.

\section{Terms of use}

This document is published under following Creative Commons-License: http://creativecommons.org/licenses/by-sa/4.0/deed.en - You may copy distribute and transmit, adapt or exhibit the work or its contents in public and alter, transform, or change this work as long as you attribute the work in the manner specified by the author or licensor. New resulting works or contents must be distributed pursuant to this license or an identical or comparable license.

By using this particular document, you accept the above-stated conditions of use.

\section{Kontakt / Contact:}

\section{peDOcs}

DIPF | Leibniz-Institut für Bildungsforschung und Bildungsinformation Informationszentrum (IZ) Bildung

E-Mail: pedocs@dipf.de

Internet: www.pedocs.de

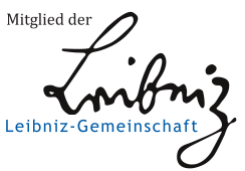




\section{von}

Lehrpersonen

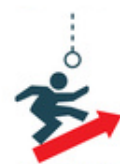

i 


\section{Impressum}

\section{Haushalt in Bildung \& Forschung (HiBiFo)}

Herausgeber:

HaBiFo Haushalt in Bildung und Forschung e.V.

Prof. ${ }^{\text {in }}$ Dr. ${ }^{\text {in }}$ Kirsten Schlegel-Matthies, Universität Paderborn

\section{Redaktion:}

Ass.-Prof. ${ }^{\text {in }}$ Dr. ${ }^{\text {in }}$ Claudia Maria Angele, Universität Wien

Werner Brandl M.A., München

Prof. ${ }^{\text {in }}$ Dr. ${ }^{\text {in }}$ Angela Häußler, PH Heidelberg

Prof. ${ }^{\text {in }}$ Dr. ${ }^{\text {in Julia Kastrup, FH Münster }}$

Prof. ${ }^{\text {in }}$ Dr. ${ }^{\text {in }}$ Kirsten Schlegel-Matthies, Universität Paderborn

Claudia Wespi lic. phil., PH Luzern

redaktion@hibifo.de•www.hibifo.de

\section{Erscheinen und Bezugsbedingungen:}

Die Zeitschrift erscheint 4 x jährlich mit einem Jahresumfang von rd. 400 Seiten (Print und Online). Abonnements verlängern sich automatisch um ein Jahr. Abonnement-Kündigungen bitte schriftlich an den Verlag. Kündigungsfrist bis drei Monate zum Jahresende.

Das digitale Angebot, alle Informationen zum Abonnement sowie zu Einzelausgaben finden Sie auf https://hibifo.budrich-journals.de.

Bestellungen bitte an den Buchhandel oder an:

Verlag Barbara Budrich GmbH, Stauffenbergstr. 7, D-51379 Leverkusen-Opladen

Tel.: +49 (0)sssss171.344.594, Fax:+49 (0)2171.344.693, info@budrich.de

www.budrich-journals.de $\bullet$ www.budrich.de

Aktuelle Mediadaten/Anzeigenpreisliste: HiBiFo 818 vom 01.02.2018

Fachliche Betreuung des Heftes: Claudia Wespi

Titelbild: Werner Brandl; Bildnachweis $(C)$ trueffelpix/fotolia

Heft 4, Jg. 8, 2019

\section{(C) 2019 Verlag Barbara Budrich GmbH Opladen • Berlin • Toronto}

Die Zeitschrift sowie alle in ihr enthaltenden Beiträge sind urheberrechtlich geschützt. Jede Verwertung, die nicht ausdrücklich vom Urheberrechtsgesetz zugelassen ist, bedarf der vorherigen Zustimmung des Verlags. Dies gilt insbesondere für Vervielfältigungen, Übersetzungen, Mikroverfilmungen und die Einspeicherung und Verarbeitung in elektronischen Systemen.

Namentlich gekennzeichnete Beiträge geben nicht in jedem Fall die Meinung der Redaktion wieder.

Druck: paper \& tinta, Warschau

Printed in Europe

ISSN 2193-8806

Online-ISSN 2196-1662 
Claudia Wespi

Editorial.....

Joanna Hellweg

Das Kohärenzgefühl als Einflussfaktor bei der Förderung

professioneller Kompetenz von Lehrpersonen im Studium 3

Werner Brandl

Professionalisierung und Professionalität - Anmerkungen

zur Bedeutung und Entwicklung von Lehrkompetenz 18

Elisabeth Eichelberger \& Susanna Holliger

SchülerInnenfirma Repair Café - Kompetenzen

entwickeln und anwenden.

Ute Bender

Videobasierte Fallanalysen als Beitrag zur Professionalisierung

von angehenden Lehrpersonen in der Ernährungs- und Verbraucherbildung -

am Beispiel der Führung von Unterrichtsgesprächen

Claudia Wespi \& Markus Steiner

„Wirtschaft entdecken“ - eine computergestützte Lernumgebung

mit Lernpotenzial für Lernende und Lehrpersonen

Silke Bartsch, Katharina Henke, Heike Müller \& Isabelle Penning

Verbraucherkompetenzen für morgen durch Lehrkräftebildung heute:

Professionalisierung von Lehrpersonen in der Verbraucherbildung 90

Sinah Gerdes \& Corinne Senn

Mit Classcraft motivierend

Wirtschaft-Arbeit - Haushalt (WAH) unterrichten 104

Jana Markert

Biografische Selbstreflexion in der Lehramtsausbildung: Erarbeitung der eigenen Bildungsbiografie mittels der systemischen Methode des Lebensfluss-Modells

Mareike Bröcheler

Rezension: Reformprojekt Care Work - Professionalisierung

der beruflichen und akademischen Ausbildung 
Bildungsbiografie

Jana Markert

Biografische Selbstreflexion in der Lehramtsausbildung: Erarbeitung der eigenen Bildungsbiografie mittels der systemischen Methode des Lebensfluss-Modells

Biografiearbeit bietet die Möglichkeit, persönliche Erfahrungen systematisch zu betrachten und dadurch eigene Erfahrungs- und Entwicklungsprozesse für sich selbst und andere sichtbar zu machen. Der Artikel beschreibt die Planung, Durchführung und Auswertung eines LehrLern-Projekts zur Erarbeitung von Bildungsbiografien Studierender im Rahmen der akademischen Lehramtsausbildung.

Schlüsselwörter: Biografische Selbstreflexion, Lehramtsstudium, Systemisches Arbeiten

Biographical self-reflection in teacher training: development of one's own educational biography using the systemic method of the life-flow model

Biographical work offers the opportunity to systematically look at personal experiences and thereby make one's own processes of experience and development visible to oneself and others. The article describes the planning, implementation, and evaluation of a teachinglearning-project for the development of educational biographies of students within the framework of academic teacher training.

Keywords: biographical self-reflection, teacher training, systemic working

\section{Theoretischer Hintergrund}

Ausgehend von den Aspekten der professionellen Handlungskompetenz von Lehrpersonen werden Biografiearbeit und deren Bedeutung im Kontext von Pädagogik vorgestellt. Danach wird die systemische Methode der Arbeit am Lebensfluss und deren potenzielle Eignung als Methode der Biografiearbeit beschrieben. Abschließend wird auf Potenzial und Grenzen reflexiven Arbeitens im Kontext der Ausbildung angehender Lehrkräfte hingewiesen. 


\subsection{Werthaltungen und Überzeugungen innerhalb der professionellen Handlungskompetenz von Lehrkräften}

Nach dem empirisch unterlegten Modell von Baumert und Kunter (2006) setzt sich professionelle Handlungskompetenz von Lehrkräften aus vier zentralen Komponenten zusammen: Professionswissen, Motivationale Orientierung, selbstregulative Fähigkeiten sowie Werthaltungen und Überzeugungen (Baumert \& Kunter, 2006). Hierbei handelt es sich um ein nicht hierarchisches Modell, d. h keiner der vier genannten Bereiche ist einem anderen übergeordnet. Vielmehr bestimmen die vier dargestellten Komponenten gleichermaßen die Ausprägung der Handlungskompetenz von Lehrkräften im Beruf. Während motivationale Orientierungen und selbstregulative Fähigkeiten eher der Eignung einer Lehrkraft zuzuschreiben sind, sind Professionswissen sowie Überzeugungen und Werthaltungen eher als Qualifikationen beschreibbar (Korneck, Kohlenberger \& Oettinghaus, 2013). Innerhalb des Komplexes von Werthaltungen und Überzeugungen unterscheiden Baumert und Kunter (2006) systematisch Wertbindungen, epistemologische Überzeugungen, subjektive Theorien über Lehren und Lernen sowie Zielsysteme voneinander. Wertbindungen orientieren sich zentral an der Berufsethik einer Profession, hier also am Ethos des Berufsbilds der Lehrerin und des Lehrers. Als Überzeugungen definieren die Autoren implizite oder explizite, subjektiv für wahr gehaltene Konzeptionen, welche die Wahrnehmung der Umwelt und das eigene Handeln beeinflussen. Dies schließt sowohl Handlungen im privaten, als auch im beruflichen Kontext ein. Aufgrund von subjektiven oder intuitiven Theorien über Lehren und Lernen kommen Lehrkräfte zur eigenständigen Entwicklung von professionellen Handlungsstrategien. Diesbezüglich gilt, „dass Lehrer, die elaborierte Subjektive Theorien besitzen, auch diejenigen sind, die professioneller handeln, da ihr Wissen in verschiedenen Bereichen reichhaltiger und differenzierter ist" (Epp, 2017, S. 43). Zielsysteme für Curriculum und Unterricht stellen präskriptive Richtungsweiser für Unterrichtsplanung und Unterrichtshandeln dar (Baumert \& Kunter, 2006). Da Überzeugungen und Werthaltungen als Qualifikationen beschrieben werden können, sind diese bereits innerhalb der akademischen Ausbildung angehender Lehrkräfte explizit zu adressieren und weiterzuentwickeln.

\subsection{Biografiearbeit}

Biografie ist „eine in einem lebenslangen Prozess erworbene Aufschichtung von Erfahrungen, die bewusst oder unbewusst [...] in unser Handeln eingehen" (Gudjons, Pieper \& Wagener, 1992, S. 16). Somit beeinflussen „Lebenserfahrungen [...] unser Denken und Handeln und nicht immer sind wir uns dieser Prägungen bewusst; der Prägungen der anderen wie auch unserer eigenen" (Cantzler, 2016, S. 6).

Biografiearbeit kann es ermöglichen, sich der Ursprünge von Prägungen, welche Einfluss auf das (private und) professionelle Denken und Handeln haben, bewusst zu 


\section{| Bildungsbiografie}

werden. Durch ihren Einsatz in unterschiedlichen Praxisfeldern erfährt die Biografiearbeit aktuell eine zunehmende Ausweitung und Diversifizierung. Oftmals, besonders in professionellen Settings, erhält sie eine strukturierte Form, innerhalb welcher die Betrachtung der Lebensgeschichte vor dem jeweiligen gesellschaftlichen Kontext im Sinne der Verarbeitung von Erlebtem und/oder der Erweiterung des zukünftigen Handlungspotenzials erfolgt. Bezogen auf das hier beschriebene Lehr-Lern-Projekt ist Biografiearbeit ebenfalls als eine strukturierte Form der Selbst- und Fremdreflexion in einem regelhaften, professionell gestalteten und intentional gesteuerten Umfeld zu verstehen. Nach Tenorth und Tippelt (2007, S. 119) hat Biografie sowohl eine subjektive Seite (individuelle Lebensgeschichte) als auch eine objektive Seite (chronologischer Lebensverlauf mit objektivierbaren Lebensereignissen). Dies führt ebenfalls Miethe (2014) unter Bezugnahme auf ein Zitat von Max Frisch („Irgendwann erfindet jeder die Geschichte, die er für sein Leben hält.") an und beschreibt die Einbettung von Erfahrungen und Erlebtem in die eigene Biografie in Abhängigkeit von individueller Relevanzzuschreibung (S. 15f.). Diese subjektive Seite von Biografie wird im hier beschriebenen Lehr-Lern-Projekt ebenfalls adressiert. Die Arbeit der Studierenden an ihren eigenen persönlichen Einstellungen, Werthaltungen und Überzeugungen ist nach Auffassung der Lehrenden ein Bestandteil der professionellen Qualifizierung angehender Lehrkräfte, wenn auch das Ergebnis dieser Arbeit (wie für andere professionalisierungsbezogene Lernprozesse ebenso zutreffend) weder für alle Lernenden gleich effektiv, noch für einen Lernenden erschöpfend möglich ist (siehe auch 1.4).

Den Mehrwert von Biografiearbeit im Kontext pädagogischen Handelns beschreibt Cantzler (2016), unter Einbeziehung von sowohl Selbst- als auch Fremdreflexion, wie folgt:

Die Auseinandersetzung mit den Lebensgeschichten anderer [...] eröffnet der pädagogischen Fachkraft ein Lernfeld, in welchem sie eigene Einstellungen und Haltungen mit denen anderer vergleicht und aufeinander abstimmt. Ursprünge und Wurzeln verschiedener Denk- und Handlungsweisen bezieht sie dabei ebenso mit ein, wie die Unterschiedlichkeit der Lebenswege und individuelle Erfahrungen. Dies öffnet ihr die Chance auf einen Perspektivwechsel, eine andere Sichtweise auf Situationen und Personen ... [und ermöglicht letztendlich] ... neue Lösungswege für zukünftiges Handeln. (Cantzler, 2016, S. 7)

Inwiefern der Beschäftigung mit der eigenen Biografie Auswirkungen auf die Entwicklung einer Person zugeschrieben werden, hat Paschelke (2013) in einem Übersichtskapitel zusammengestellt und daraus u. a folgende vorgesehenen Erträge identifiziert: Verbesserung der Selbstkenntnis, Förderung der Selbstreflexion und wahrnehmung (von Pädagogen bzw. Lehrern), Förderung der Selbsttätigkeit, Förderung der Verstehens- und Interpretationskompetenz, Perspektivwechsel sowie das Bewusstsein für das Problem der Ungleichheit im Erziehungsverhältnis (S. 63). Der potenziell konstruktive Nutzen für die Qualifikation von Lehrkräften ist deutlich 
erkennbar, wenn auch bisher innerhalb der erziehungswissenschaftlichen Forschung selten konkretisiert (ebd.) und damit bisher wenig empirisch belegt.

\subsection{Systemisches Arbeiten}

Nach Ruhe (2014, S. 36) existiert für die Biografiearbeit kein geschlossenes Methodensystem. Vielmehr fokussiert sie Methoden, welche auch in anderen Kontexten nutzbar sind, auf die Lebensgeschichte von Menschen. Das hier beschriebene LehrLern-Projekt wendet eine Methode aus dem systemischen Arbeiten auf die Erarbeitung der Bildungsbiografie an.

Nach systemischem Verständnis ist der Mensch immer zugleich als biologisches und als soziales Wesen zu betrachten. Die systemische Perspektive rückt deshalb die dynamische Wechselwirkung zwischen den biologischen und psychischen Eigenschaften einerseits und den sozialen Bedingungen des Lebens andererseits ins Zentrum der Betrachtung, [...]. (Rotthaus, o. J.)

Als System kann eine beliebige Gruppe von Elementen bezeichnet werden, die durch Beziehungen miteinander verbunden und durch eine Grenze von ihren Umwelten abgrenzbar sind (von Schlippe \& Schweitzer, 2013, S. 31). Jeder Mensch bewegt sich zeitgleich innerhalb mehrerer, voneinander verschiedener Systeme. Die unterschiedlichen Elemente eines Systems stehen miteinander in Beziehung und haben Auswirkungen auf die jeweiligen Handlungsmöglichkeiten eines Menschen und damit auf sein gezeigtes Verhalten. Dabei sind für das systemische Arbeiten nicht die objektiven Gegebenheiten des Systems von Bedeutung. Vielmehr entsteht das relevante System erst durch die subjektiven Bedeutungszuschreibungen der agierenden Personen. Sämtliche Arbeitsformen, die diesen systemischen Blick im Sinne eines erkenntnistheoretischen Ansatzes benutzen, können als systemische Praxis oder systemisches Arbeiten beschrieben werden (ebd.).

Die Methode der Lebensfluss-Arbeit kann dazu dienen, eine (mehr oder weniger konkrete) Zielfrage zu beantworten. Dabei bedient sie sich der systematischen Betrachtung eines Lebensabschnitts der fragenden Person. Durch die fragende Person werden auf dem Fußboden entlang eines Therapieseils, welches den zu betrachtenden Lebensabschnitt darstellt, relevante Ereignisse chronologisch dargestellt (bspw. durch Bilder, Symbole, Worte). Hierbei handelt es sich i. d. R. um eine stille und geschützte Arbeit, welche von dezentem Nachfragen begleitet werden kann. Auf diese Weise entsteht der Lebensfluss als begehbares Bild. Das Bewegen innerhalb des Lebensflussbilds ermöglicht eine vielfältige und intensive Auseinandersetzung mit dem betrachteten Lebensabschnitt unter dem Aspekt der Zielfrage. 


\subsection{Selbstreflexion in der Lehramtsausbildung}

Seit den 1980er Jahren werden die Begriffe ,Reflexion' und ,kritische Reflexion' im Kontext der Lehrkräftebildung immer häufiger verwendet (Berndt, Häcker \& Leonhard, 2017, S. 9). In den 1990er Jahren vollzieht sich mit dem sog. ,reflective turn' die deutliche Abkehr von der Rolle der Lehrperson als rein wissensvermittelnde Persönlichkeit. Vielmehr erweitert sich das Berufsbild um spezifische professionsbezogene Kompetenzen, welche notwendig sind, die komplexen Strukturen von Unterricht zu handhaben. Die Reflexion des eigenen professionellen Handelns erhält dadurch in der schulpraktischen Tätigkeit, jedoch v. a. in der Lehramtsausbildung, eine zunehmende Bedeutung. Der „Einübung eines erfahrungsbezogenen Reflexionsvermögens" wird ein hohes Potenzial für Veränderungen im Lehrerhandeln zugeschrieben (Bolland, 2011, S. 26). Neuß (2009) geht sogar noch weiter, indem er die Reflexion des eigenen Lernweges als eine Voraussetzung dafür bezeichnet, das Lernen anderer zu fördern, anzuleiten und zu verstehen (S. 85). Bereits 2001 empfahl auch der Wissenschaftsrat reflektives Berufswissen in die universitäre Lehramtsausbildung zu integrieren (S. 41). Diese Empfehlung wurde 2004 von der Kultusministerkonferenz (KMK) der Länder übernommen. Die KMK führt in ihren Standards für die Ausbildung von Lehrpersonen den Einsatz biografisch-reflektiver Methoden an, um $u$. a die eigenen biografischen Lernerfahrungen zu analysieren und zu reflektieren (KMK, 2004/2019, S. 6). Selten wird in der Literatur auf Grenzen oder gar auf Risiken des (häufigen) Einsatzes von Reflexion in der Lehrkräfteprofessionalisierung hingewiesen. So führt Häcker (2017) die Begrenztheit des menschlichen Vermögens das eigene Ich zu betrachten an (S. 23) und weist auf die Möglichkeit der Entstehung von Formen defensiven Reflektierens hin (S. 24).

\section{Ziele und Fragestellungen des Projekts}

Im hier beschriebenen Lehr-Lern-Projekt sollen der Einsatz der Bildungsbiografie in der Lehramtsausbildung sowie die Umsetzbarkeit der systemischen Methode der Lebensfluss-Arbeit in einer Gruppe von Lehramtsstudierenden exemplarisch untersucht werden. Daraus ergeben sich zum einen folgende Lehr- und Lernziele, zum anderen sollen nachfolgende Forschungsfragen beantwortet werden:

\subsection{Lehrziele}

\section{Den Studierenden soll}

- ein (erfahrbarer) Zugang zur eigenen Bildungsbiografie ermöglicht werden.

- Raum gegeben werden, um auf einer persönlichen Ebene lernförderliche sowie Lernen erschwerende Faktoren analysieren und reflektieren zu können.

- die Betrachtung verschiedener Bildungsbiografien ermöglicht werden. 
Bildungsbiografie |

\subsection{Lernziele}

Die Studierenden

- betrachten Bildung aus unterschiedlichen biografischen Perspektiven.

- analysieren ihre erarbeiteten Ergebnisse im Hinblick auf den Umgang mit lernförderlichen und -hinderlichen Faktoren.

- stellen ihre Ergebnisse denen ihrer Mitstudierenden gegenüber (Kleingruppe).

\subsection{Fragestellungen}

Die übergeordnete Fragestellung des Projekts lautet: Welchen Erkenntnisgewinn erlangen Studierende durch die Erarbeitung von Bildungsbiografien mittels des Einsatzes der systemischen Methode des Lebensfluss-Modells innerhalb des institutionellen Rahmens eines Hochschulseminars?

Daraus ergeben sich folgende zu klärende Unterfragen:

- Inwiefern nutzen die Studierenden das Lernangebot?

- Welche übergeordneten Erkenntnisse ziehen die Studierenden aus der eigenen Biografiearbeit und aus der ihrer Mitstudierenden?

- Wie bewerten die Studierenden die angebotene Lerngelegenheit?

\section{Methodisches Vorgehen, Projektumsetzung}

Das hier vorgestellte Lehr-Lern-Projekt adressiert angehende Lehrkräfte der Sonderpädagogik mit dem Kernfach Wirtschaft-Technik-Haushalt/Soziales (WTH). Die Biografiearbeit wurde im Wintersemester 2017/2018 innerhalb des Moduls ,Einführung in die Fachdidaktik WTH' mit Studierenden des 5. Fachsemesters durchgeführt. In der ersten Semesterwoche wurde die Rolle der schulischen Lehrperson für das Unterrichtsgeschehen thematisiert, u. a über Baumert und Kunter (2006), Tulodziecki, Herzig und Blömeke (2017) und Helmke (2017), sowie das Thema Biografiearbeit vorgestellt. Die konkreten Lehrveranstaltungen zur Bildungsbiografie (zwei Seminartage, je 15:00-19:00 Uhr) erfolgten in der zweiten und dritten Semesterwoche.

Die systemische Methode der Arbeit am Lebensfluss stellt eine Möglichkeit der intensiven Auseinandersetzung mit eigenen biografischen Erfahrungen dar (siehe 1.3). Die chronologische Strukturierung und klare Benennung von einzelnen Ereignissen spricht zunächst die kognitive Ebene der Lernenden an. Soweit es zugelassen wird, kann die Bildungsbiografie durch die Lebensfluss-Methode ebenso emotional erlebbar werden. Die Lernenden haben die Möglichkeit, sich innerhalb der eigenen Bildungsbiografie real zu bewegen. Selbstgewählte symbolische Darstellungen, das 


\section{Bildungsbiografie}

Abschreiten im individuellen Tempo, das Einnehmen unterschiedlicher Betrachtungsperspektiven, die stille Reflexion von Lernereignissen zum Nachempfinden und zur Prüfung können die Auseinandersetzung vertiefen. Die Zielfrage für die Biografiearbeit lautete: „Wie hat sich für Sie ,Lernen' im Laufe ihres bisherigen Lebens gestaltet?“

\subsection{Vorbereitung der Studierenden}

Für die Einführung in das Thema „Biografiearbeit' sowie um Grundzüge einer systemischen Haltung zu verdeutlichen, konnte an das Seminar ,Kooperative Beratung' angeknüpft werden. Dieses vermittelt Elemente der kollegialen Supervision basierend auf Mutzeck (2005). Die dort gelehrten Grundregeln und -techniken einer wertschätzenden Kommunikation liegen ebenfalls dem systemischen Arbeiten zugrunde. Die Studierenden der Sonderpädagogik belegten das Seminar ,Kooperative Beratung' innerhalb des Diagnostik-Moduls ihres ersten Förderschwerpunkts.

Vorbereitend auf die Arbeit an der Biografie wurde die Haltung gegenüber der biografisch arbeitenden Person gemäß Reich (2008) thematisiert: „Der Lehrende nimmt beim biografischen Arbeiten eine interessierte, zugewendete Haltung gegenüber dem Erinnernden ein und bringt sich nur gegebenenfalls durch sanfte Fragen [...] ein. Ansonsten hält er sich eher im Hintergrund und vertritt die Position des Zuhörenden." Daran anknüpfend wurden Arbeitsregeln besprochen:

- Freiwilligkeit des Gezeigten und Gesagten, Beachtung von Grenzen

- Schweigepflicht aller Beteiligten, Vertraulichkeit der Arbeitssituation

- Wertungsfreiheit und wertschätzende Kommunikation

\subsection{Erarbeitungsphase}

\subsubsection{Erster Seminartag}

Der erste Seminartag begann mit der Wiederaufnahme der Themen Biografiearbeit und Systemisches Arbeiten. Es wurden die Arbeitsregeln wiederholt (siehe 3.1) und die Methode der Arbeit am Lebensfluss ausführlich erläutert sowie praktisch demonstriert. Dazu erfolgte eine 45-minütige Demonstration im Plenum. Überraschender- und zugleich erfreulicherweise fand sich hierfür relativ zügig ein Studierender als Freiwilliger. Die Lehrende ist durch die Ausbildung zur systemischen Beraterin mit der Arbeit am Lebensfluss gut vertraut. Sie hat diese Methode bereits mehrfach und zu verschiedenen Themen angeleitet sowie innerhalb ihrer Ausbildung selbst erfahren dürfen. Somit ist sie in deren Anwendung versiert. Sie weiß an der Methode zu schätzen, dass sowohl ein stilles, als auch ein intensiv dialogisch begleitetes Arbeiten möglich sind. Während einer stillen Arbeit ist manchmal von außen kaum zu erkennen, an welcher Stelle der Erarbeitungsprozess sich gerade befindet. Zeitgleich 
kann für die arbeitende Person jedoch eine intensive Auseinandersetzung erfolgen. Diese Eigenschaft der Lebensflussarbeit ermöglicht es den Studierenden, selbstverantwortlich das für sie jeweils richtige $\mathrm{Ma}$ an Öffnung und Schutz zu bestimmen. Nach der Demonstration wurden methodische Rückfragen an die Lehrende zugelassen, und das demonstrierte systemische Fragen nochmals ausführlich besprochen. Um die Ergebnisse inhaltlich zu reflektieren, erhielten die Studierenden einen schriftlichen Arbeitsauftrag, mit welchem sie sich direkt im Anschluss an die eigene Biografiearbeit in Einzelarbeit auseinandersetzten.

Biografiearbeit erfordert eine offene Haltung der erinnernden Person, um die notwendige selbsttätige Handlung zu initiieren und dadurch die bewusste Auseinandersetzung überhaupt erst zu ermöglichen. Besonders im Rahmen intentionaler Lernprozesse kann dies eine Herausforderung darstellen. Deshalb wurde die konkrete Einwahl in die fünf Kleingruppen den Studierenden selbst überlassen. Lediglich der Studierende, welcher bereits in der Demonstration seine Bildungsbiografie erarbeitet hatte, musste in einer 5er-Gruppe als weitere beobachtende Person mitarbeiten. Damit ergab sich pro Kleingruppe folgende Rollenverteilung:

- eine Person, welche am Lebensfluss die eigene Bildungsbiografie erarbeitet

- eine Person, welche die Arbeit am Lebensfluss begleitet und unterstützt

- eine beobachtende Person mit der Aufgabe der Zeitüberwachung

- eine weitere Person, welche selbst als letzte biografisch gearbeitet hat

Die Rollenverteilung wurde nochmals ausführlich besprochen sowie ein Arbeitsblatt mit einem Leitfaden für die Arbeit am Lebensfluss ausgegeben. Jede Kleingruppe arbeitete in einem extra vorbereiteten Raum. Durch ein Hinweisschild an der Tür (,Beratung. Bitte nicht stören.') konnten Unterbrechungen der Arbeit vermieden werden. Die Lehrende wechselte während der Gruppenarbeitsphase zwischen den einzelnen Kleingruppen (i. d. R. einmal pro Gruppe innerhalb der 45 Minuten), um das Arbeiten zu hospitieren und die Einhaltung des Zeitplans zu unterstützen. Am Ende des ersten Seminartags trafen sich die Lernenden im Plenum wieder, um sich über die Kleingruppenarbeit auszutauschen und den Seminartag abzuschließen.

\subsubsection{Zweiter Seminartag}

Zu Beginn des zweiten Seminartags erfolgte zunächst die inhaltliche Wiederaufnahme des Themas. Die Arbeitsregeln (siehe 3.1) wurden wiederholt und die Arbeitsfähigkeit der Kleingruppen wiederhergestellt. Danach wurde zeitnah die Arbeit in den am ersten Seminartag gebildeten Kleingruppen wiederaufgenommen. Nach jeder Erarbeitungsphase erfolgte eine 15-minütige Pause, in welcher die Studierenden ihre gesammelten Eindrücke sortieren konnten. Durch die rasche Abfolge der Arbeitsphasen am zweiten Tag war die Einhaltung der 15-minütigen Pausen besonders wichtig. Die Studierenden wurden angehalten, in den Pausen unbedingt den Arbeits- 


\section{Bildungsbiografie}

raum (und nach Möglichkeit auch das Gebäude) zu verlassen, um im Freien ihren Gedanken Raum zu geben.

\subsection{Ergebnissicherung}

Alle Studierenden wurden angehalten, ihre persönlichen Ergebnisse mithilfe des Arbeitsauftrags ,Einzelarbeit' festzuhalten. Die Lehrende eröffnete die Möglichkeit von Konsultationen bei noch offenen oder neu entstandenen Fragen.

Die Ergebnissicherung im Plenum erfolgte am Ende des zweiten Seminartags nach der letzten Kleingruppenarbeit. An dieser Stelle wurde zunächst ein methodisches Feedback eingeholt. Danach erfolgte die Sammlung der Ergebnisse zu Lernressourcen und Lernherausforderungen. Die Studierenden waren angehalten, weiterhin die Anonymität ihrer Mitstudierenden zu wahren und gewonnene Eindrücke ohne Nennung von Klarnamen und in Form von Ich-Formulierungen zu äußern.

\subsection{Datenauswertung}

Um die übergeordneten Erkenntnisse der Studierenden zu erfassen (siehe Forschungsfragen unter 2.3) wurde auf der Lernplattform Moodle 2.0 innerhalb des Online-Kurses zum Modul ,Einführung in die Fachdidaktik WTH' eine kurze, anonyme Befragung zur subjektiven Bewertung der im Seminar umgesetzten Biografiearbeit durchgeführt. Die fünf erfragten Items können der Tabelle 1 entnommen werden. Aufgrund der geringen Stichprobengröße und der kleinen Anzahl an erfragten Items wurde weder für die quantitative noch für die qualitative Auswertung eine Statistiksoftware verwendet. Die deskriptive Auswertung der Onlinebefragung in Form der prozentualen und absoluten Antworthäufigkeiten ist im Befragungstool der Lernplattform Moodle2.0 bereits integriert. Die qualitative Auswertung der Freitextantworten erfolgte fallübergreifend als beschreibende Analyse orientiert an der inhaltlich strukturierenden Inhaltsanalyse nach Kuckartz (2018, S. 97ff.). Die Bildung der thematischen Kategorien erfolgte induktiv, dabei bildeten ein einzelner Satz bzw. eine komplette Freitextantwort die kleinste bzw. größte Analyseeinheit.

\section{Ergebnisse des Lehr-Lern-Projekts}

Die Anzahl der im Wintersemester 2017/2018 im Modul ,Einführung in die Fachdidaktik WTH' eingeschriebenen Studierenden betrug $N=25$. Obwohl es sich bei der akademischen Ausbildung für das Lehramt Sonderpädagogik um ein Präsenzstudium handelt, besteht in keiner Lehrveranstaltung eine Anwesenheitspflicht. Die Möglichkeit der Erarbeitung der eigenen Bildungsbiografie nahmen $n=21$ Studierenden war. Davon haben alle die online gestellte Kurzumfrage (fünf Items, Tab. 1) beantwortet. 
Tab. 1: Inhalt der Studierendenumfrage (Online-Kurzumfrage) (Quelle: eigene Darstellung)

\begin{tabular}{|c|c|c|}
\hline Item & Formulierung Item & Antwortformat \\
\hline 1 & $\begin{array}{l}\text { Die Betrachtung der eigenen Lernbiografie } \\
\text { ist für angehende Lehrkräfte sinnvoll. }\end{array}$ & $\begin{array}{l}\text { vierstufige Likert-Skala (Zustimmung) } \\
\text { und Freitext (Begründung der Antwort) }\end{array}$ \\
\hline 2 & $\begin{array}{l}\text { Die Betrachtung meiner eigenen Lernbiogra- } \\
\text { fie war für mich persönlich erkenntnisreich. }\end{array}$ & vierstufige Likert-Skala (Zustimmung) \\
\hline 3 & $\begin{array}{l}\text { Die Betrachtung meiner eigenen Lernbiogra- } \\
\text { fie war für mich als angehende Lehrkraft } \\
\text { erkenntnisreich. }\end{array}$ & vierstufige Likert-Skala (Zustimmung) \\
\hline 4 & $\begin{array}{l}\text { Meine aus der Biografiearbeit gewonnenen } \\
\text { Erkenntnisse können bei der Gestaltung von } \\
\text { Lernsituationen (für SuS) hilfreich sein. }\end{array}$ & vierstufige Likert-Skala (Zustimmung) \\
\hline 5 & $\begin{array}{l}\text { Die angebotene Form der Biografiearbeit } \\
\text { war für den Rahmen akademische ,Lehr- } \\
\text { amtsausbildung' passend. }\end{array}$ & $\begin{array}{l}\text { vierstufige Likert-Skala (Zustimmung) } \\
\text { und Freitext (Begründung der Antwort) }\end{array}$ \\
\hline
\end{tabular}

\subsection{Was die Studierenden mitgenommen haben}

\subsubsection{Ressourcen und Herausforderungen innerhalb der eigenen Bildungsbiografie}

Die konkreten Ergebnisse zu Lernressourcen und Lernherausforderungen wurden wie unter 3.3 beschrieben gesammelt. Die herausgearbeiteten Einzelaspekte konnten $\mathrm{zu}$ vier strukturell verschiedenen Bereichen zusammengefasst werden (Institution Schule, Peers und Familie, Lernende/Lernender, Lehrkraft). 81 \% der Studierenden stimmten der Aussage, persönliche Erkenntnisse gewonnen zu haben (Item 2) voll $(n=5)$ bzw. eher zu $(n=12) ; 19 \%$ stimmten dem weniger $(n=3)$ bzw. gar nicht zu $(\mathrm{n}=1)$. In Bezug auf die Gewinnung von Erkenntnissen als angehende Lehrkraft (Item 3) stimmten der Aussage $86 \%$ der Befragten voll $(\mathrm{n}=8)$ bzw. eher $\mathrm{zu}$ $(\mathrm{n}=10) ; 14 \%(\mathrm{n}=3)$ stimmten dem weniger zu. Dennoch verteilte sich die $\mathrm{Zu}-$ stimmung zur Aussage, dass gewonnene Erkenntnisse bei der Gestaltung von Lernsituationen hilfreich sein können (Item 4) folgendermaßen: $95 \%$ stimmten voll $(\mathrm{n}=12)$ bzw. eher $(\mathrm{n}=8)$ zu und $5 \%(\mathrm{n}=1)$ stimmten weniger $\mathrm{zu}$. Die unterschiedliche Verteilung der Antworten innerhalb der Items 3 und 4 könnte mit der Möglichkeit der Einsichtnahme in die Bildungsbiografien Mitstudierender erklärt werden.

\subsubsection{Biografiearbeit in der akademischen Lehramtsausbildung}

Der Aussage, dass die Betrachtung der eigenen Lernbiografie für angehende Lehrkräfte sinnvoll ist, stimmten $67 \%$ der Befragten voll $(\mathrm{n}=14)$ und $33 \%$ eher zu 


\section{Bildungsbiografie}

$(n=7)$. Dabei begründeten die Studierenden ihre Zustimmung inhaltlich unterschiedlich:

(1) Die Sinnhaftigkeit bestehe darin, Einflussfaktoren auf das Lernen wahrnehmen und eigenes (zukünftiges) berufliches Handeln entsprechend gestalten zu können (Kategorie Inhalt). Bsp.: „Man sieht, wo es Schwierigkeiten [...] gab und kann diese auf das eigene Lehrerverhalten beziehen (z.B. ungerechte Behandlung durch Lehrkräfte in Schulzeit $\rightarrow$ anders handeln als Lehrperson).“

(2) Die Rolle der Lehrkraft an sich kann wahrgenommen und die eigene Ausgestaltung dieser Rolle analysiert werden (Kategorie Haltung). Bsp.: „Diese Erkenntnisse sind, glaube ich, sehr hilfreich, um ein genaueres Bild davon zu bekommen, was für einen eine gute Lehrperson ausmacht [...]. So bekommt man auch eine Idee davon, welche Werte und welches Menschenbild man später als Lehrkraft selbst vertreten will, $[\ldots]$. .

(3) Der Switch vom Fokus auf Lehren (Lehrkraft) zum Fokus auf Lernen (Schülerinnen und Schüler) kann vollzogen werden (Kategorie Perspektivwechsel). Bsp.: „Da man empathisch nachvollziehen kann, in welcher Situation sich Schülerinnen und Schüler befinden, zumal Lehrkräfte eher aufs Lehren fokussiert sind.“"

(4) Die Entstehung eigener Überzeugungen und Werthaltungen bezüglich Lehren und Lernen kann betrachtet und analysiert werden (Kategorie Überzeugungen). Bsp.: „Ich denke es ist sinnvoll, weil wir vieles bewusst oder unterbewusst aus unserer eigenen Schulzeit oder von einzelnen Lernorten mitnehmen.“

Die Studierenden halten die Betrachtung der eigenen Bildungsbiografie mehrheitlich für sinnvoll. Sie begründen dies zum einen über konkrete Inhalte und die Möglichkeit des Perspektivwechsels, zum anderen über den eigenen Qualifikationsanspruch (Bsp. Arbeit an der professionellen Haltung). Dies deckt sich mit den Ergebnissen anderer Untersuchungen zur Biografiearbeit im Hochschulkontext. Vogt schreibt bereits 1996 über biografische Lernanregungen, dass sie „neben der methodischen und inhaltlichen Fachkompetenz die Notwendigkeit der Persönlichkeitsbildung des zukünftigen [...] Pädagogen betonen.“(S. 183).

\subsection{Systemisches Arbeiten im Kontext Hochschullehre}

Das intensive Arbeiten in den Kleingruppen an beiden Seminartagen sowie die ausführlichen Freitext-Antworten in der Onlineumfrage zeugen davon, dass die Studierenden die angebotene Lernform intensiv genutzt und für sich bearbeitet haben.

Der Aussage, dass die angebotene Form der Biografiearbeit für den Rahmen der akademischen Lehramtsausbildung passend war (Item 5), stimmten $57 \%$ der Befragten voll $(\mathrm{n}=12), 38 \%$ eher $(\mathrm{n}=8)$ und $5 \%$ weniger $\mathrm{zu}(\mathrm{n}=1)$. In der Begründung ihrer Antwort führten die Studierenden folgendes an:

(1) Eine praxisorientierte Methode kennengelernt zu haben, welche auf die Lernenden aktivierend wirkt und durch die Möglichkeit der Selbsterfahrung die persön- 
liche Entwicklung adressiert (Kategorie Methode). Bsp.: „Aktivierend, spannend, kommunikativ."

(2) Die gelungene Umsetzung bzw. Aufbereitung der Methode durch bspw. den Wechsel der Sozialformen (Einzelarbeit, Kleingruppe, Plenum) oder den passenden Grad an Selbstorganisation (Kategorie Seminar). Bsp.: „Gut, dass in freiwillig gewählten Kleingruppen gearbeitet wurde [...]. Gut, dass genügend Zeit eingeplant wurde. Gute Vorbereitung [der Räume].“

(3) Limitierende Faktoren wie ungenügend Zeit für die Kleingruppenarbeit, ungeschulte Begleitpersonen, Aufteilung der Biografiearbeit auf zwei Seminartage anstatt eines langen Tages oder die Notwendigkeit einer Vertrauensbasis wurden als potenzielle Schwierigkeiten benannt (Kategorie Limitationen). Bsp.: ,[...] war nicht ausgebildet, passende Fragen zu stellen. [...] schöner, mit externen/ausgebildeten Personen zu arbeiten." Hier ist weiterhin zu ergänzen, dass bereits während des methodischen Feedbacks am Ende des zweiten Seminartags die Studierenden Unsicherheiten äußerten, passende Fragen formuliert zu haben. Außerdem beschrieben sie Schwierigkeiten, die vorgegebene Zeit von maximal 45 Minuten für eine Biografiearbeit einzuhalten.

(4) Keine Begründung der Antwort bzw. eine inhaltlich eher auf Item 1 passende Antwort (Kategorie Null). Bsp.: „Die Biografiearbeit war passend, da es für die Entwicklung der eigenen Lehrperson/-persönlichkeit von großer Bedeutung ist [...].“

Zusammenfassend konnte gezeigt werden, dass die Methode der Lebensflussarbeit eine im Kontext Hochschullehre umsetzbare Lehr-Lern-Form darstellt. Die Studierenden schätzen sowohl die Methode an sich, als auch deren konkrete Umsetzung im hier beschriebenen Projekt positiv ein. Die genannten Limitationen sollten bewusst adressiert und mit den Lernenden offen kommuniziert werden, um dennoch innerhalb des institutionellen Rahmens von Hochschullehre reflexive Lernformen anbieten zu können.

\section{Fazit}

Das hier beschriebene Lehr-Lern-Projekt ist als systematisch reflektierte Praxis mit dem Ziel der Weiterentwicklung akademischer Lehramtsausbildung in Anlehnung an das Konzept des Scholarship of Teaching and Learning (SoTL) (Huber, 2014, S. 21) mit Bezug zur Aktionsforschung (Altrichter, Posch \& Spann, 2018, S. 11) zu verstehen. Trotz der empirischen Überprüfung des Einsatzes der Lebensflussarbeit in Kombination mit Biografiearbeit liefert der Artikel aufgrund des geringen Stichprobenumfangs sowie des Fehlens einer Kontrollgruppe lediglich Hinweise (anstelle von belastbaren Ergebnissen) für die Gestaltung von Hochschullehre in der akademischen Lehramtsausbildung. Durch den Einsatz von Audio- oder Videografie während der Ergebnissicherung am Ende des zweiten Seminartags würde eine breitere Daten- 


\section{Bildungsbiografie}

basis für die qualitative Auswertung zur Verfügung stehen. Hier hätten u. U. Dynamiken zwischen Selbst- und Fremdreflexion aufgezeigt werden können.

Die für das Projekt definierten studierendenbezogenen Lernziele der Reflexion der eigenen Bildungsbiografie, der Betrachtung von Bildungswegen aus unterschiedlichen Perspektiven durch die intensive Arbeit in Kleingruppen und das Formulieren relevanter Einflussfaktoren auf Lernprozesse wurden erreicht. Es ist gelungen, den Studierenden mithilfe der Methode der Lebensflussarbeit einen erfahrbaren Zugang zur eigenen Bildungsbiografie zu ermöglichen und lernförderliche sowie Lernen erschwerende Faktoren auf einer persönlichen Ebene zu analysieren und zu reflektieren. Unter Beachtung der beschriebenen Limitationen des Projekts kann die Aussage getätigt werden, dass die Projektteilnehmenden die angebotene Form der Selbst- und Fremdreflexion zu einem sehr hohen Prozentsatz gut bzw. sehr gut angenommen haben. Dies legt die Vermutung nahe, dass auch bei anderen Studierenden mit einer hohen bzw. sehr hohen Akzeptanz für diese Lehrform zu rechnen ist.

Die tatsächliche Effektivität dieser Methode in Bezug auf die Professionalisierung angehender Lehrkräfte sollte in weiteren möglichst repräsentativen Studien überprüft werden. Mögliche Weiterentwicklungen der hier vorgestellten Arbeit könnten in Form von spezifischen Themenbiografien (Bsp. Essbiografie, Konsumbiografie) thematisch erfolgen. Weiterhin kann Biografiearbeit punktuell eingesetzt werden, um Aufmerksamkeit und/oder Motivation für ein Thema zu wecken und damit einen didaktischen Ansatzpunkt für die Erarbeitung eines Lerngegenstands bieten.

\section{Literatur}

Altrichter, H., Posch, P. \& Spann, H. (2018). Lehrerinnen und Lehrer erforschen ihren Unterricht (5. Aufl.). Bad Heilbrunn: Julius Klinkhardt.

Baumert, J. \& Kunter, M. (2006). Stichwort: Professionelle Kompetenz von Lehrkräften. Zeitschrift für Erziehungswissenschaft, 9(4), 469-520.

Berndt, C., Häcker, Th. \& Leonhard, T. (Hrsg.). (2017). Reflexive Lehrerbildung revisited. Traditionen - Zugänge - Perspektiven. Bad Heilbrunn: Julius Klinkhardt.

Bolland, A. (2011). Forschendes und biografisches Lernen. Das Modellprojekt Forschungswerkstatt in der Lehrerbildung. Bad Heilbrunn: Verlag Julius Klinkhardt.

Cantzler, A. (2016). Spurensuche: Wie Biographiearbeit pädagogisches Handeln ändert. Betrifft Kinder, 10, 6-11.

Epp, A. (2017). Von der Schule in die Berufsausbildung. Soziale Konstruktionen durch Lehrkräfte über ungünstige Faktoren in der Bildungsbiografie von Schülerinnen und Schülern. Opladen: Verlag Barbara Budrich. 
Gudjons, H., Pieper, M. \& Wagener, B. (1992). Auf meinen Spuren. Das Entdecken der eigenen Lebensgeschichte. Hamburg: Bergmann und Helbig.

Häcker, T. (2017). Grundlagen und Implikationen der Forderung nach Förderung von Reflexivität in der Lehrerinnen- und Lehrerbildung. In C. Berndt, Th. Häcker \& T. Leonhard (Hrsg.), Reflexive Lehrerbildung revisited. TraditionenZugänge-Perspektiven (S. 21-46). Bad Heilbrunn: Julius Kinkhardt.

Helmke, A. (2017). Unterrichtsqualität und Lehrerprofessionalität. Diagnose, Evaluation und Verbesserung des Unterrichts (7. Aufl.). Seelze: Klett-Kallmeyer.

Huber, L. (2014). Scholarship of Teaching and Learning: Konzept, Geschichte, Formen, Entwicklungsaufgaben. In L. Huber, A. Pilniok, R. Sethe, B. Szczyrba \& M. Vogel (Hrsg.), Forschendes Lehren im eigenen Fach. Scholarship of Teaching and Learning in Beispielen (S. 19-36). Bielefeld: Bertelsmann.

Korneck, F., Kohlenberger, M. \& Oettinghaus, L. (2013). Lehrerüberzeugungen und Unterrichtshandeln im Fach Physik. PhyDid B - Didaktik der Physik-Beiträge zur DPG-Frühjahrstagung.

http://www.phydid.de/index.php/phydid-b/article/view/472

Kuckartz, U. (2018). Qualitative Inhaltsanalyse. Methoden, Praxis, Computerunterstützung (4. Aufl.). Weinheim: Beltz Juventa.

Kultusministerkonferenz (KMK). (2004/2019). Standards für die Lehrerbildung: Bildungswissenschaften. https://www.kmk.org/fileadmin/Dateien/veroeffentlichungen_beschluesse/2004/ 2004_12_16-Standards-Lehrerbildung-Bildungswissenschaften.pdf

Miethe, I. (2014). Biografiearbeit. Lehr- und Handbuch für Studium und Praxis (2. Aufl.). Weinheim: Beltz.

Mutzeck, W. (2005). Kooperative Beratung (5. Aufl.). Weinheim: Beltz.

Neuß, N. (2009). Biographisch bedeutsames Lernen. Empirische Studien über Lerngeschichten in der Lehrerbildung. Studien zur Bildungsgangforschung, Band 25. Opladen: Budrich.

Paschelke, S. (2013). Biographie als Gegenstand von pädagogischer Forschung und Arbeit. Möglichkeiten einer konstruktiven pädagogischen Biographiearbeit. Bad Heilbrunn: Julius Klinkhardt.

Reich, K. (2008). Biografiearbeit. In K. Reich (Hrsg.), Methodenpool. $\mathrm{http}: / /$ methodenpool.uni-koeln.de/uebersicht.html

Rotthaus, W. (o. J.) für Deutsche Gesellschaft für Systemische Therapie, Beratung und Familientherapie (DGSF) e. V. (2019). Was heißt systemisch? Grundsätzliches zum systemischen Arbeiten. https://www.dgsf.org/service/was-heisstsystemisch

Ruhe, H.G. (2014). Praxishandbuch Biografiearbeit. Methoden, Themen und Felder. Weinheim: Beltz Juventa. 


\section{Bildungsbiografie}

von Schlippe, A. \& Schweitzer, J. (2013). Lehrbuch der systemischen Therapie und Beratung I. Das Grundlagenwissen (2. Aufl.). Göttingen: Vandenhoeck \& Ruprecht.

Tenorth, H.-E. \& Tippelt, R. (Hrsg.). (2007). Beltz Lexikon Pädagogik. Weinheim: Beltz.

Tulodziecki, G., Herzig, B. \& Blömeke, S. (2017). Gestaltung von Unterricht. Bad Heilbrunn: Julius Klinkhardt.

Vogt, A. (1996). Lernen in lebensgeschichtlichen Bezügen - Biographisches Lernen und Lehren in der Hochschule. In W. Schulz (Hrsg.), Lebensgeschichten und Lernwege (S. 176-184). Hohengehren: Schneider.

Wissenschaftsrat. (2001). Empfehlungen zur künftigen Struktur der Lehrerbildung. http://www.wissenschaftsrat.de/download/archiv/5065-01.pdf

\section{Verfasserin}

Prof. ${ }^{\text {in }}$ Dr. ${ }^{\text {in }}$ Jana Markert

Universität Leipzig, Erziehungswissenschaftliche Fakultät

Institut für Förderpädagogik

Marschnerstraße 29 e

D-04109 Leipzig

E-Mail: Jana.Markert@uni-leipzig.de

Internet: https://www.erzwiss.uni-leipzig.de/wirtschaft-technik-haushalt-soziales 